\title{
Thermal Image Enhancement Algorithm Based on Adaptive Fusion Technique of Multi Color Space
}

\author{
Rafid A. Haleot ${ }^{1}$, Ziad M. Abood ${ }^{2}$ and Ghada S. Karam ${ }^{2}$ \\ ${ }^{1-2}$ College of Education/ Mustansiriyah University \\ ${ }^{3}$ Mustansiriyah University, Baghdad \\ Iraq
}

\begin{abstract}
This paper presents an improvement of the enhancement algorithm of thermal images in order to increase the quality of low contrast and low Illumination Infrared images. Our approach based on Fusion of Multi-Color Spaces, two main stages are tested: in the first stage the thermal images mapped from RGB color space to LAB and HSV Color Spaces where enhancement is done and reconverted to the RGB space, the luminance component (V) in HSV Color Space and the luminance component (L) in LAB Color Space are enhance using CLAHE method. In the second stage, the contrast improving is done by the fusing of both results. The results of the experimental demonstrate the performance of the proposed algorithm for improving the quality of thermal images in comparison with the published works.
\end{abstract}

Keywords: Enhancement of Infrared Image, CLAHE, LAB color space, HSV Color Space, Image Fusion.

\section{INTRODUCTION}

Thermal rays cannot be seen by human eyes, the imaging system in thermal spectral enable passively receive thermal rays and convert into thermal images. It has been vastly used in domains like military, clinical imaging, manufacturing, and by the surveillance [1-7] Images in the thermal spectral compared with images in the visible light spectral suffer from several ingrained disadvantage, one of that disadvantage is depressed contrast of objective to background, which produce it complicated to understand objects from the background. [8-11]. Hence, the enhancement of contrast is one of essential pre-processing to progress the quality of thermal images [12].

The classification of Enhancement methods are: frequency domain and spatial methods [13]. The principal bunch utilizes spatial space image preparing which legitimately controls the pixels. Numerous spatial image improvement strategies depend on examination and modification of histogram; while different techniques depend on the transformation of neighborhood contrast [14]. The most traditional enhancement technique of image is the histogram equalization (HE). (HE) is a worldwide preparing method, so the whole tone of the image has been varied such as more brilliant or dim image. In much circumstances, these techniques broaden the dynamic range of the images in nearby areas, resulting to more distinguish tonal modify in the image.

The another area which depend on using frequency area techniques by modifying and control the frequency substance of the image. These approach of the enhancement depend on using frequency change or transformation, like, DCT, Fourier, and so forth. Now and again some properties of image, for example, low and high-recurrence coefficient histograms might be so firmly stuffed that distinguish them from each other might be inconceivable [14,15].

To improve differentiates in images the Adaptive histogram equalization (AHE) has been used [16, 17]. A versatile rendition of this calculation called CLAHE (contrast limited adaptive histogram equalization) [17, 18]. This methodology is fiercely utilized for improving the neighborhood differentiate or contrast for thermal image.

The CLAHE enhancement algorithm effective in deferent model such as RGB model, Lab model, and HSI model. The Lab color model can be presented by different three components which is lightness, blue-yellow, and components of green-red. This designed color model was to be perceptually comfortable to the humans color vision $[19,20]$. The color model HSV is otherwise called the HSL color model. This model like Lab color model incorporates three components: saturation, hue, and value (lightness) [21]. This is an elective portrayal of the RGB color [22]. The HSV color model was designed to add color encoding to 
get monochrome broadcasts, permitting existing recipients to obtained new color broadcasts without alteration and modification, as the luminance signal is communicated unmodified [23].

The rest of this paper is sorted out as follows. Section 2 presented the proposed method. Section 3 presented the results of experimental and discussion. At last, the conclusions from this research are stated in Section 4.

\section{PROPOSED METHOD}

The proposed thermal image enhancement algorithm is presented in the flow process below and block diagram in Figure 1.

\section{General steps Algorithm}

1) Input Thermal Image.

2) Convert RGB image to HSV model.

3) Convert RGB image to LAB model

4) Enhance the luminance component (V) in HSV model using CLAHE.

5) Enhance the luminance component (L) in LAB model using CLAHE.

6) Convert from HSV to RGB model.

7) Convert from LAB to RGB model.

8) Fusion (HSV to RGB) image with (LAB to RGB) image.

9) Display the enhanced image.

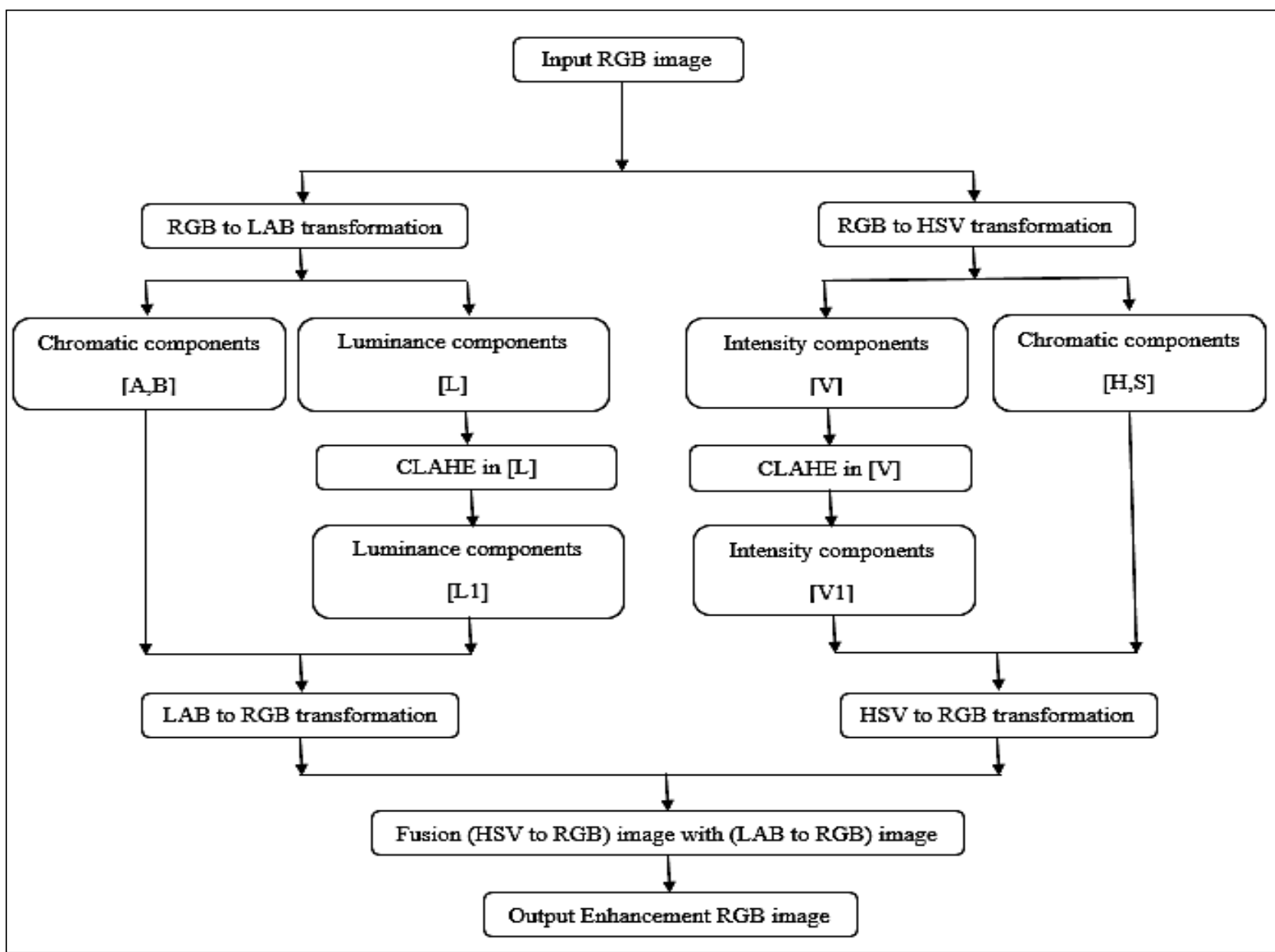

Figure 1. Block Diagram of the algorithm of Thermal Image Enhancement

\section{COLOR SPACES}

In digital image, RGB color space is consists independent images was three components (red (R), green (G), and blue (B)). The HSV color space is intended from the prime RGB by nonlinear transformation [24]. HSV is setup on the theory of human visual observation and is appropriate for deciphering and portraying shading. So it is a lot more like a people impression of shading, this model depicts the shading space as far as chromatic information: hue (H), saturation (S), and achromatic information: value (V) components. The force part is the weighted normal of three shading channels and less touchy to noise. HSV color space was best frequently has use application-oriented space. 


$$
\begin{aligned}
S & =\frac{\max (R, G, B)-\min (R, G, B)}{\max (R, G, B)} \\
V & =\max (R, G, B) .
\end{aligned}
$$

Lab color space is molded with L (Lightness cannel) and (a, b) (chromatic segments). This shading space can communicate a more extensive shading extent or range than RGB and HSV because it includes all perceivable colors and that means its gamut exceeds than RGB color model [25]. One of the most important attributes of the Lab color space is the device independency, so is used as an interchange format between different devices [26].

$$
\begin{aligned}
& L^{*}=116 f\left(\frac{Y}{Y_{n}}\right)-16 \\
& a^{*}=1500\left(f\left(\frac{X}{X_{n}}\right)-f\left(\frac{Y}{Y_{n}}\right)\right) \\
& b^{*}=200\left(f\left(\frac{Y}{Y_{n}}\right)-f\left(\frac{Z}{Z_{n}}\right)\right)
\end{aligned}
$$

\section{LUMINANCE COMPONENT ENHANCEMENT}

$\mathrm{V}$ component (in HSV model) and the L component (in LAB model) were enhancement adopted by CLAHE. The V and L components are divided to $8 \times 8$ tiles. The clip-limit applied is 0.02 . We applied Uniform distribution as the histogram frame for the tiles the image. The modified gray levels expression for standard CLAHE technique with Uniform Distribution can be given as [27]:

Where $R_{\max }=$ maximum pixel value

$$
R=\left[R_{\max }-R_{\min }\right] \times P(f)+R_{\min }
$$

$\mathrm{R}_{\min }=$ minimum pixel value

$\mathrm{R}=$ comuted pixel value

$\mathrm{p}(\mathrm{f})=$ cummulative probability distribution

\section{PERFORMANCE EVALUATION}

There are many procedures presented as a quality measurement of the image enhancement technology [28]. The (EME) image enhancement measure is utilize to measure the quality and adopt the optimal processing parameters of images which introduced by Agaian [29]:

$$
E M E_{m 1, m 2}=\max \left(\frac{1}{m 1 . m 2} \sum_{t=1}^{m 1} \sum_{k=1}^{m 2} 20 \cdot \log \frac{X_{\max , k, t}^{w}}{X_{\min , k, t}^{w}}\right) \ldots .
$$

Where $X_{\text {max }, k, t}^{w}=$ maximum of the image $\mathrm{x}(\mathrm{n}, \mathrm{m})$ inside the block $w_{k, t}$

$X_{\mathrm{min}, k, t}^{w}=$ minimum of the image $\mathrm{x}(\mathrm{n}, \mathrm{m})$ inside the block $w_{k, t}$.

\section{EXPERIMENTAL RESULTS}

The developed algorithm (illustrated in section 2) was implemented in MATLAB 7.4.1 on the LTIR v1.0 dataset [30]. This dataset include 20 series of thermal infrared image (car, street, trees, horse, garden et al.). System performance was evaluated using EME measurement. Figure 2 the results of thermal image enhancement demonstrate respectively by several method:

a- Original image.

b- The histogram equalization method.

c- The CLAHE method.

d- The proposed method to enhance the image.

The analysis display that the proposed method demonstrate more specifics in the gained enhanced thermal images, so it gives preferred visual quality over the histogram adjustment method and CLAHE.

Table 1 shows experimental results for lowest EME of original images, and apply processing of proposed algorithm. It is noted that the results of the quality gained by the proposed algorithm is that it best concerning EME measurement. 


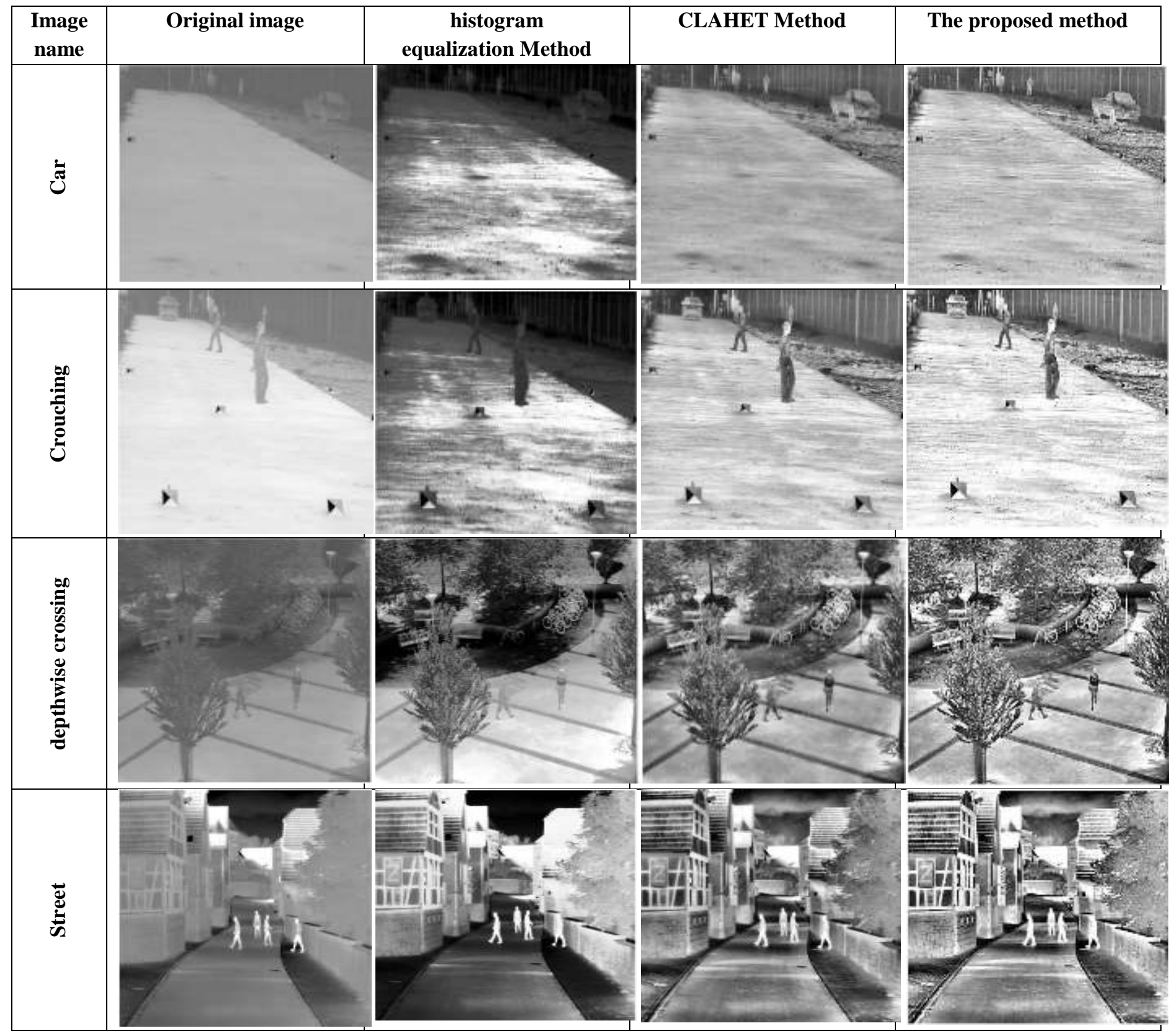

Fig. 2. Thermal Image Enhancement in current study

Table (1) The results of EME measures values of medical image enhancement

\begin{tabular}{ccccc}
\hline Images & Original & $\begin{array}{c}\text { Histogram } \\
\text { Equalization }\end{array}$ & CLAHE & $\begin{array}{c}\text { Proposed } \\
\text { Method }\end{array}$ \\
\hline Car & 0.871 & 8.653 & 4.029 & 17.64 \\
crouching & 1.420 & 9.833 & 5.676 & 14.530 \\
Depth wise crossing & 4.240 & 18.044 & 17.140 & 29.951 \\
Street & 5.3170 & 10.5149 & 14.9738 & 19.289 \\
\hline
\end{tabular}

\section{CONCLUSION}

We present an approach for Enhancement the Thermal Image Based on adaptive Fusion technique of Multi Color Space. This Algorithm for enhancement the Thermal Image enable obtain more detailed and contrast thermal image with the following properties: brightness gradient and unequal illumination. The improvement results proposed are favorably compared with other modern methods. Proposed algorithm has proven useful in implementations of thermal imaging. 


\section{ACKNOWLEDGEMENTS}

The authors would like to thank Mustansiriyah University for supported (https://uomustansiriyah.edu.iq/) Baghdad- Iraq for its support in this work.

\section{REFERENCES}

1. T. Grulois, G. Druart, N. Guérineau, A. Crastes, H. Sauer, and P. Chavel, "Extra-thin infrared camera for low-cost surveillance applications", Opt. Lett. Vol. 39, pp. 3169-3172, 2014.

2. B. Prasad, K. Prabha, and P. Kumar, "Condition monitoring of turning process using infrared thermography technique-An experimental approach”, Infrared Phys. Technol. Vol. 81, pp. 137-147 2017.

3. B. Lahiri, S. Bagavathiappan, T. Jayakumar, and J. Philip, "Medical applications of infrared thermography: a review", Infrared Phys. Technol. Vol. 55, pp. 221-235, 2012.

4. Y. Gao, J. Ma, and A. L. Yuille, "Semi-supervised sparse representation based classification for face recognition with insufficient labeled samples”, IEEE Trans. Image Process. Vol. 26, pp. 2545-2560, 2017.

5. J. Ma, J. Jiang, C. Liu, and Y. Li, "Feature guided Gaussian mixture model with semi-supervised EM and local geometric constraint for retinal image registration”, Inform. Sci. Vol. 417, pp. 128-142, 2017.

6. S. Bagavathiappan, B. B. Lahiri, T. Saravanan, J. Philip, and T. Jayakumar, "Infrared thermography for condition monitoringa review", Infrared Phys. Technol. Vol. 60, pp. 35-55, 2013.

7. J. Ma, J. Zhao, J. Tian, A. L. Yuille, and Z. Tu, "Robust point matching via vector field consensus", IEEE Trans. Image Process. Vol. 23, pp. 1706-1721, 2014.

8. A. Rogalski, "Recent progress in infrared detector technologies", Infrared Phys. Technol. Vol., pp. 136-154, 2011.

9. J. H. Kim, J. H. Kim, S. W. Jung, C. K. Noh, and S. J. Ko, "Novel contrast enhancement scheme for infrared image using detail-preserving stretchin", Opt. Eng. Vol. 50, p. 077002, 2011.

10. J. Ma, J. Zhao, Y. Ma, and J. Tian, "Non-rigid visible and infrared face registration via regularized Gaussian fields criterion", Pattern Recogn. Vol. 48, pp. 772-784, 2015.

11. J. Ma, C. Chen, C. Li, and J. Huang, "Infrared and visible image fusion via gradient transfer and total variation minimization", Inform. Fusion, Vol. 31, pp. 100-109, 2016.

12. R. Lai, Y. T. Yang, B. J. Wang, and H. X. Zhou, “A quantitative measure based infrared image enhancement algorithm using plateau histogram”, Opt. Commun. Vol. 283, pp. 4283-4288, 2010.

13. K. Pratt William, "Digital Image Processing", John Wiley \& Sons, 3 rd edition, 2001.

14. A. Grigoryan and S. Agaian, "Image enhancement", in Advances in Imaging and Electron Physics. New York: Academic, pp. 165-243, 2004.

15. A. Jain, "Fundamentals of Digital Image Processing", Englewood Cliffs, NJ: Prentice-Hall, 1989.

16. J. A. Stark, "Adaptive image contrast enhancement using generalizations of histogram equalization", Image Processing, IEEE Transactions on, Vol. 9, no. 5, pp. 889-896, 2000.

17. S. M. Pizer, E. P. Amburn, J. D. Austin, “Adaptive Histogram Equalization and Its Variations”, Computer Vision, Graphics, and Image Processing 39, vol. 355-368, 1987.

18. K. Zuiderveld, "Contrast Limited Adaptive Histogram Equalization”, In: P. Heckbert: Graphics Gems IV, Academic Press, 1994

19. Hunter, Richard Sewall, "Photoelectric color-difference meter". Josa. Proceedings of the winter meeting of the optical society of America, Vol. 38, no.7, p. 661. 2000.

20. Hunter, Richard Sewall, "Accuracy, precision, and stability of new photo-electric color-difference meter". Josa Proceedings of the thirty-third annual meeting of the optical society of America, Vol. 38, no. 12, p. 1094. 2001.

21. M. C. Jobin Christ, R. M. S. Parvathi, "Segmentation of Medical Image using Clustering and Watershed Algorithms", American Journal of Applied Sciences, Vol. 8, no. 12, pp. 1349-1352, 2011.

22. G. Stockman and L. Shapiro, "Computer Vision", Prentice Hall, 2001.

23. S. Sural, G. Qian, and S. Pramanik, "Segmentation and histogram generation using the HSV color space for image retrieval, "presented at IEEE International Conference on Image Processing, Rochester, New York, 2002.

24. Jinxiang Ma, "Contrast Limited Adaptive Histogram Equalization-Based Fusion in YIQ and HSI Color Spaces for Underwater Image Enhancement”, International Journal of Pattern Recognition and Articial Intelligence Vol. 32, no. 7, p.89, 2018.

25. Ziad M. Abood Ghada S. Karam Rafid. E. Haleot Enhancement Human Face Recognition Based on Fusing of Infrared and Visible Images. Conference 23, College of Education, 26-27 April 2015, Special Issue, no. 2, pp.73-79, 2017.

26. Edgar Chavolla, Daniel Zaldivar, Erik Cuevas and Marco A. Perez, Color Spaces Advantages and Disadvantages in Image Color Clustering Segmentation, Chapter in Studies in Computational Intelligence Springer International Publishing AG Learning in Image Processing, Studies in Computational Intelligence 730, https, pp.3-22, 2018. doi.org/10.1007/978-3-319-63754-9-1. 
27. Sos. S. Agaian, "Visual morphology", Proceedings of SPIE, Nonlinear Image Processing X, San Jose, CA, vol. 3646, pp. 139-150, 1999.

28. Sos S. Agaian, Karen P. Lentz, and Artyom M. Grigoryan, “A New Measure of Image Enhancement”, IASTED International Conference on Signal Processing \& Communication, pp. 19-22, 2000.

29. A. Silva Eric, Panetta Karen, Sos S. Agaian, "Quantifying imagesimilarity using measure of enhancement by entropy", Proc. SPIE 6579, Mobile Multimedia/Image Processing for Military and Security Applications 2007, vol. 65790U, 2007.

30. A. Berg and J. Ahlberg and M. Felsberg, "A Thermal Object Tracking Benchmark", Advanced Video and Signal Based Surveillance, AVSS, $12^{\text {th }}$ IEEE International Conference on, 2015.

\section{Biographies}

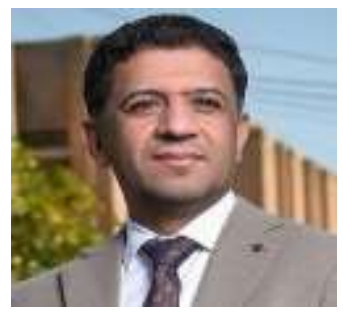

Rafid Aedan Haleot received the bachelor from University of Baghdad, Master's degrees and the Ph.D. degree from from Mustansiriyah University, in 2017. He has nearly 15 years of experience in teaching in computer science department. He is currently a lecturer with the Department of Computer Science, College of Education, Mustansiriyah University. He has published over 8 articles in international and national journals and conferences. His fields of interests include image processing.

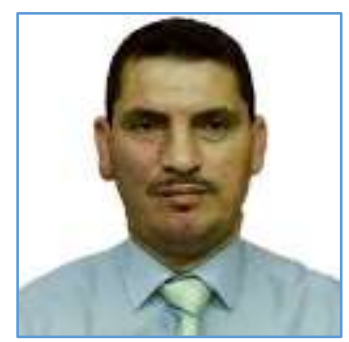

Ziad M. Abood received the bachelor,1994, and Ph.D.,2006 degree from Mustansiriyah University,Bagdad-Iraq, and the Master degree from Yarmouk University,Jordan in 2001. He has nearly 27 years of experience in teaching with image processing and computer Physics science, College of Education, Mustansiriyah University with Scientific Degree Professor. He has published over 80 articles in international and national journals and conferences, and30 Books.

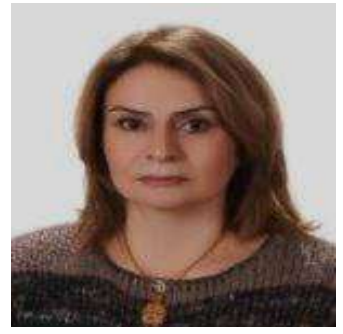

Ghada S. Karam received the bachelor, Master and Ph.D. degree from Mustansiriyah University, in 2005. She has nearly 25 years of experience in teaching in Physics science department,Optics, College of Education, Mustansiriyah University with Scientific Degree Professor. Has published over 25 articles in international and national journals and conferences. 